The 2014 Annual Conference of the International Society for Environmental Information Sciences (ISEIS)

The 2014 Atlantic Symposium of the Canadian Association on Water Quality (CAWQ)

The 2014 Annual General Meeting and 3oth Anniversary Celebration of the Canadian Society for Civil Engineering Newfoundland and Labrador Section (CSCE-NL)

The and International Conference of Coastal Biotechnology (ICCB) of the Chinese Society of Marine Biotechnology and Chinese Academy of Sciences (CAS)

\title{
Research on Passive Contaminant Transport in a Vegetated Channel
}

\author{
Fatima Jahra ${ }^{1, *}$ and Yoshihisa Kawahara ${ }^{2}$ \\ ${ }^{1}$ Oceanic Consulting Corporation, A1B 2X5, St. John's, Canada \\ ${ }^{2}$ Propessor, Department of Civil and Environment Engineering, Hiroshima University, 739-8527, Higashi- \\ Hiroshima, Hiroshima, Japan \\ *Corresponding author's e-mail: jahra.fatima@oceaniccorp.com
}

\begin{abstract}
Vegetation plays an important role in the physical, ecological, and hydraulic functions of streams, rivers and many other water bodies and can affect the transport of water, sediment and nutrients both within the channel and to or between the riparian zones. This research studied the momentum and passive contaminant transport mechanism in a vegetated channel with a floodplain. Three dimensional numerical simulation and physical experimentations were conducted for turbulent flow in the presence of model vegetation. A non-linear $k-\varepsilon$ model with a vegetation model for turbulent flow and an algebraic flux model (DH model) for contaminant transport were adopted. An in-house code developed by the authors was implemented for numerical simulations. Model vegetation zones were prepared in the channel to predict the mixing mechanism. The numerical results were compared with corresponding experimental observations. A good agreement between the simulated and experimental results was observed in terms of pollutant concentration.
\end{abstract}

Keywords: Passive contaminant transport, non-linear $k-\varepsilon$ model, algebraic flux model, vegetated channel.

\section{Introduction}

Rivers and streams usually have vegetation in close proximity. Vegetation can occupy nearly every geomorphic position within the fluvial environment (Figure 1). The presence of floodplain vegetation significantly affects the flow field in the main channel and hence mass and momentum exchange between the main channel and the floodplain. Vegetation can affect the water quality by transporting sediment, and nutrients both within the channel and to or between the riparian zones. In recent years solute transport in rivers has become an important topic in environmental hydraulics. Thus, there has been an increasing interest in predicting solute transport processes in compound channel flows for controlling pollution levels in rivers (Lin and Shiono, 1995). Numerical simulation as well as physical experiments on flow distribution and momentum transport in the presence of vegetation has been studied by many researchers in the last three decades e.g. Arnold et al. (1985), Shimizu and Tsujimoto (1994), Naot et al. (1996), Rameshwaran and Shiono (2007), Kang et al. (2009), Sanjou et al. (2010), Jahra (2011). The impact of vegetation on pollutant or sediment transport is still an on-going research topic.

The compound channel flows are characterized by complicated flow structure due to the presence of a shear layer at the interface between the main channel and the floodplain. The presence of vegetation makes the flow pattern more complicated. The additional drag exerted by vegetation reduces the mean velocity, turbulence intensities and bed shear stress within a 
Joint Conferences:

The 2014 Annual Conference of the International Society for Environmental Information Sciences (ISEIS)

The 2014 Atlantic Symposium of the Canadian Association on Water Quality (CAWQ)

The 2014 Annual General Meeting and zoth Anniversary Celebration of the Canadian Society for Civil Engineering Newfoundland and Labrador Section (CSCE-NL)

The 2nd International Conference of Coastal Biotechnology (ICCB) of the Chinese Society of Marine Biotechnology and Chinese Academy of Sciences (CAS)

vegetated zone. This baffling promotes solute transport, sediment deposition and suppresses bed erosion. Arnold et al. (1985) measured the dye concentration in a compound channel and deduced the lateral mixing coefficient of the tracer. Wood and Liang (1989) conducted laboratory experiments to measure the tracer concentration and developed a two-dimensional semi-analytical model to predict tracer concentration in the open channel. Jaque and Ball (1995) experimentally studied the mixing of pollutant concentrations in a compound channel. Lin \& Shiono (1995) performed numerical simulation using a linear and a non-linear $k-\varepsilon$ models and concluded that the tracer concentration predicted by the non-linear $k$ - $\varepsilon$ model gives better agreement than that of the linear $k-\varepsilon$ model. Shiono et al. (2003) carried out experiments and numerical simulation with a non-linear $k-\varepsilon$ model, and an algebraic stress model to predict the solute transport with an eddy diffusivity model in an asymmetric compound channel. Kang et al. (2009) investigated the characteristics of solute transport in a rectangular open channel with submerged vegetation.

Until now the characteristics of passive contaminant transport has not been discussed in detail for different types of vegetation arrangement in compound channels. Thus, the need to predict the flow and transport of solute (pollutant), sediment and nutrients in the presence of vegetation, leads to laboratory experiments and numerical computations. The objective of present research is to investigate the characteristics of pollutant transport in a channel with a floodplain with different vegetation layouts through physical experiments and numerical computations. Numerical computations were conducted by a non-linear $k-\varepsilon$ model with a vegetation model, coupled with a scalar flux model.

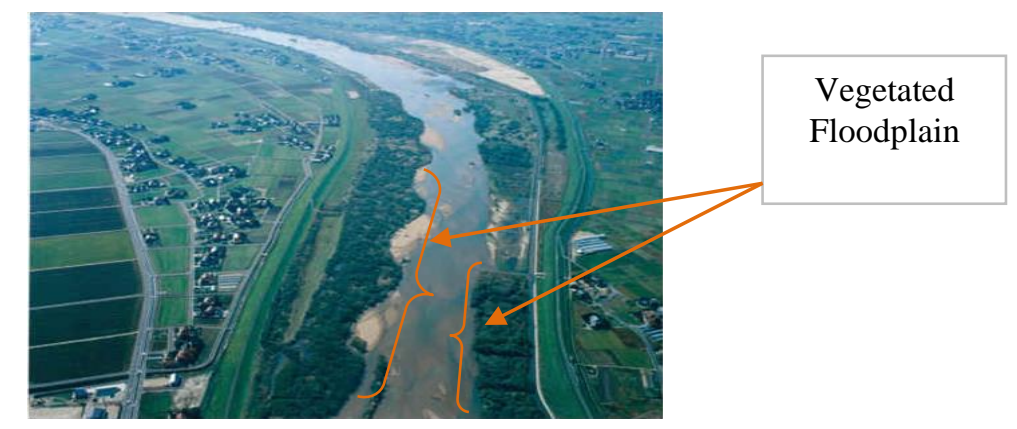

Figure 1. Photo of a natural river with vegetation

\section{Experimental Setup}

The flow and solute measurements in a channel with a floodplain (compound channel) were conducted by Jahra et al. (2011). Experiments were carried out in The Hydraulic Engineering Laboratory of Hiroshima University, Japan. The length, width and slope of the experimental flume are 2,200 cm, $182 \mathrm{~cm}$ and 1/500, respectively. The length-width ratio is 1:12. The floodplain was constructed on either side of the channel, having a height of $4 \mathrm{~cm}$ and a width of $45.5 \mathrm{~cm}$. The vegetation was idealized with wooden rigid cylinders of $0.3 \mathrm{~cm}$ diameter and $5 \mathrm{~cm}$ height. The measurements were carried out with two types of vegetation zones prepared over one side of the floodplain as shown in Figure 2 with (a) Case A1 - the floodplain fully covered by model vegetation; and Case A2 - a $10 \mathrm{~cm}$ wide vegetation belt located along the junction of the main channel and the floodplain. Cross sectional layouts of the two types of vegetation and the flume photo are shown in Figure 3 and Figure 4, respectively. Three mean velocity components 


\section{The 2014 International Conference on Marine and Freshwater Environments}

\section{Joint Conferences:}

The 2014 Annual Conference of the International Society for Environmental Information Sciences (ISEIS)

The 2014 Atlantic Symposium of the Canadian Association on Water Quality (CAWQ)

The 2014 Annual General Meeting and zoth Anniversary Celebration of the Canadian Society for Civil Engineering Newfoundland and Labrador Section (CSCE-NL)

The and International Conference of Coastal Biotechnology (ICCB) of the Chinese Society of Marine Biotechnology and Chinese Academy of Sciences (CAS)

were measured by two-component electromagnetic current meters (both L-type and I-type). Sodium Chloride $(\mathrm{NaCl})$ was used as tracer and its concentration was measured by a densitometer with a probe diameter of $0.2 \mathrm{~cm}$. $\mathrm{NaCl}$ was injected continuously at $x=900 \mathrm{~cm}$ and at several locations y (noted in Table 1) with a $0.3 \mathrm{~cm}$ diameter nozzle. The discharge in the flume was $Q=30 \mathrm{~L} . \mathrm{s}^{-1}$. The Froude number is 0.07 for Case A1 and 0.08 for Case A2 (considering flume width and mean flow velocity). The initial concentration of solute was $1 \%$. Solute was dissolved in water and diluted by alcohol to set the injected mixture density the same as the flume water density at the working temperature. The red dotted lines in Figure 2 indicate the measurement sections. Table 1 demonstrates the injection locations and the experimental conditions.

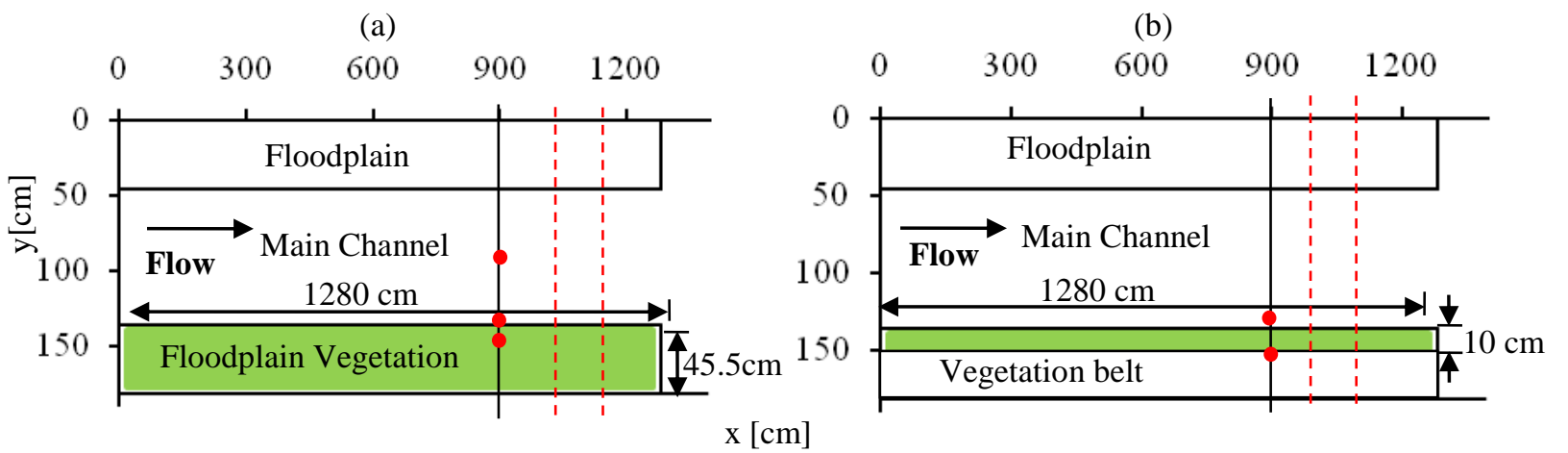

Figure 2. Vegetation placement and solution injection points (plan view) for (a) floodplain fully covered by model vegetation (Case A1); (b) $10 \mathrm{~cm}$ wide vegetation belt located along the junction of the main channel and the floodplain (Case A2). The red dot shows the locations of the injection points in the y-direction.

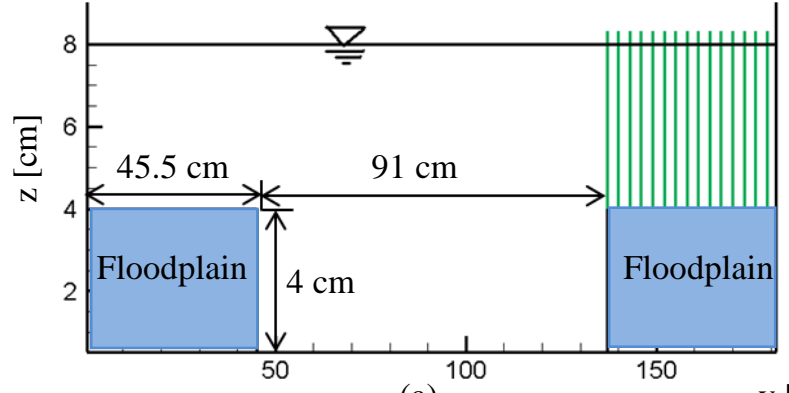

(a)

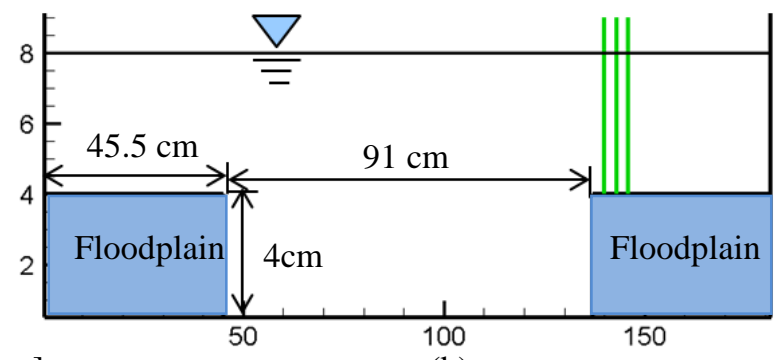

(b)

Figure 3. Vegetation placements in the experimental flume with (a) floodplain fully covered by vegetation (Case A1); (b)floodplain vegetation belt (Case A2).

Table 1. Experimental condition for solute transport

\begin{tabular}{|c|c|c|c|c|c|c|}
\hline \multirow{2}{*}{ Test Case } & \multirow{2}{*}{$\begin{array}{c}\text { Sub Test } \\
\text { Case }\end{array}$} & \multirow{2}{*}{$\begin{array}{l}\text { Water depth } \\
{[\mathrm{cm}]}\end{array}$} & \multicolumn{3}{|c|}{ Solute injection location } & \multirow{2}{*}{ Injection flow rate $\left[\mathrm{mL} . \mathrm{s}^{-1}\right]$} \\
\hline & & & $\mathrm{x}[\mathrm{cm}]$ & $\mathrm{y}[\mathrm{cm}]$ & $\mathrm{z}[\mathrm{cm}]$ & \\
\hline \multirow{3}{*}{ A1 } & S1 & \multirow{3}{*}{8.0} & \multirow{3}{*}{900} & 91 & \multirow{3}{*}{7.9} & \multirow{3}{*}{3.0} \\
\hline & $\mathrm{S} 2$ & & & 136 & & \\
\hline & S3 & & & 138 & & \\
\hline A2 & S4 & 7.9 & 900 & 136 & 7.8 & 3.0 \\
\hline
\end{tabular}




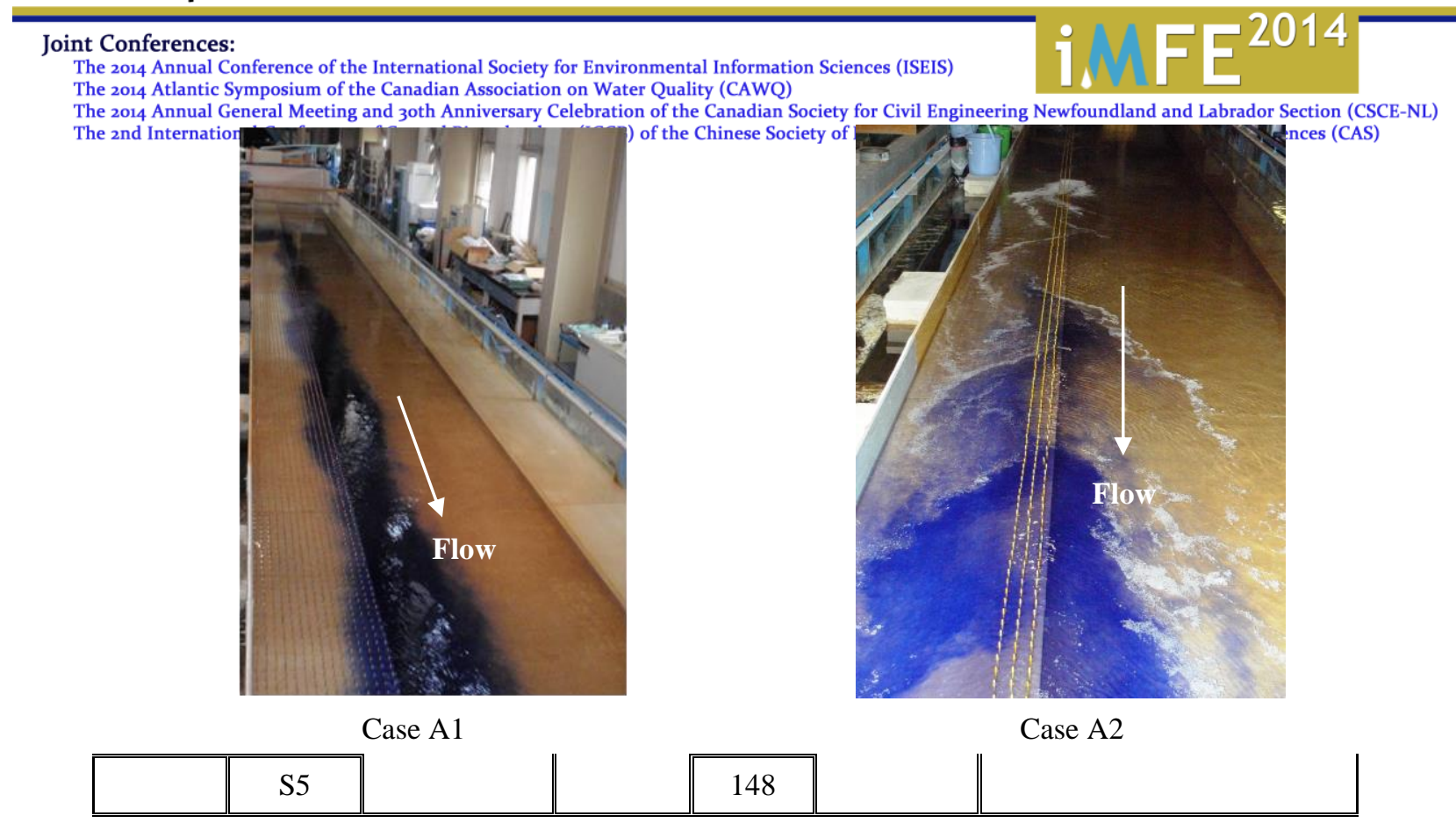

Figure 4. Photograph of dye spreading in the experimental flume.

\section{Mathematical Formulations}

\section{Turbulence Model}

The double averaged continuity and momentum equations were used for the numerical computation of turbulent flows with vegetation zones (Jahra, 2011).

$$
\begin{aligned}
& \frac{\partial\left\langle\overline{u_{i}}\right\rangle}{\partial x_{i}}=0 \\
& \frac{\partial\left\langle\overline{u_{i}}\right\rangle}{\partial t}+\frac{\partial\left\langle\overline{u_{i}}\right\rangle\left\langle\overline{u_{j}}\right\rangle}{\partial x_{j}}+\frac{\partial}{\partial x_{j}}\left(\left\langle\overline{u_{i}{ }^{\prime} u_{j}}{ }^{\prime}\right\rangle+\left\langle{\overline{u_{i}}}^{\prime \prime}{\overline{u_{j}}}^{\prime \prime}\right\rangle\right)=g_{i}-\frac{1}{\rho} \frac{\partial\langle\bar{p}\rangle}{\partial x_{i}}+v \frac{\partial^{2}\left\langle\overline{u_{i}}\right\rangle}{\partial x_{j} \partial x_{j}}-\frac{1}{\rho} \frac{\partial\left\langle\bar{p}^{\prime \prime}\right\rangle}{\partial x_{i}}+v\left\langle\frac{\partial^{2} \bar{u}_{i}}{\partial x_{j} \partial x_{j}}\right\rangle
\end{aligned}
$$

Here, the over bar stands for time averaged, single prime for the deviation from the time average, angle brackets for the spatial average, and double primes for the deviation from the spatial average. The last two terms on the right hand side of momentum equation representing the pressure and viscous drag forces are modeled as follows:

$$
-\frac{1}{\rho} \frac{\partial\langle\bar{p} "\rangle}{\partial x_{i}}+v\left\langle\frac{\partial^{2} \bar{u}_{i}}{\partial x_{j} \partial x_{j}}\right\rangle=-\frac{1}{2} C_{D} \lambda U_{i} \sqrt{U_{j} U_{j}}=-F_{i}
$$

$C_{D}$ is the drag coefficient $(=1.1)$ and $\lambda(=0.033)$ is the vegetation density defined by $\lambda=D / l_{x} l_{y} . D$ is the diameter of the vegetation stem and $l_{x}$ and $l_{y}$ are the adjacent vegetation distances in the $x$ and $y$ directions, respectively. Hereafter we use upper cases for double-averaged velocity as $\left\langle\overline{u_{i}}\right\rangle=U_{i}$ for brevity. The Reynolds stress term and the velocity correlation term in the momentum equation are modeled as:

$$
-\left\langle\overline{u_{i} u_{j}^{\prime}}+\overline{u_{i}} \overline{u_{j}}\right\rangle \equiv-\overline{u_{i} u_{j}}=v_{t} S_{i j}-\frac{2}{3} k \delta_{i j}-\frac{k}{\varepsilon} v_{t} \sum_{\beta=1}^{3} C_{\beta}\left(S_{\beta i j}-\frac{1}{3} S_{\beta \alpha \alpha} \delta_{i j}\right)
$$




\section{The 2014 International Conference on Marine and Freshwater Environments}

Joint Conferences:

The 2014 Annual Conference of the International Society for Environmental Information Sciences (ISEIS)

The 2014 Atlantic Symposium of the Canadian Association on Water Quality (CAWQ)

The 2014 Annual General Meeting and 3oth Anniversary Celebration of the Canadian Society for Civil Engineering Newfoundland and Labrador Section (CSCE-NL)

The and International Conference of Coastal Biotechnology (ICCB) of the Chinese Society of Marine Biotechnology and Chinese Academy of Sciences (CAS)

Where, $v_{t}$ is the eddy viscosity and $k$ and $\varepsilon$ are the double-averaged turbulent kinetic energy and its dissipation rate, respectively. $C_{\mu}$ has been computed using the formula proposed by Kimura $\&$ Hosoda (2003). A detail extression of the above equation can be found in the reference Jahra, 2011.

The transport equations for $k$ and $\varepsilon$ are written as:

$$
\begin{aligned}
& \frac{\partial k}{\partial t}+\frac{\partial U_{j} k}{\partial x_{j}}=\frac{\partial}{\partial x_{m}}\left[\left(\frac{v_{t}}{\sigma_{k}}+v\right) \frac{\partial k}{\partial x_{m}}\right]+P_{r o d}+S_{k}-\varepsilon \\
& \frac{\partial \varepsilon}{\partial t}+\frac{\partial U_{j} \varepsilon}{\partial x_{j}}=\frac{\partial}{\partial x_{m}}\left[\left(\frac{v_{t}}{\sigma_{\varepsilon}}+v\right) \frac{\partial \varepsilon}{\partial x_{m}}\right]+\frac{\varepsilon}{k} C_{\varepsilon 1} P_{r o d}+S_{\varepsilon}-C_{\varepsilon 2} \frac{\varepsilon^{2}}{k} \\
& P_{r o d}=-\overline{u_{i} u_{j}} \frac{\partial U_{i}}{\partial x_{j}}
\end{aligned}
$$

The double-averaging concept introduces source and sink terms, $S_{k}$ and $S_{\varepsilon}$, into the transport equations for $k$ and $\varepsilon$, which was originally proposed by Green (1992) and modified by the authors (Jahra et al. 2010).

$$
\begin{aligned}
& S_{k}=F_{i} U_{i}-2 C_{D} \lambda \sqrt{U_{i} U_{i}} \\
& S_{\varepsilon}=\frac{3}{2} \frac{\varepsilon}{k} F_{i} U_{i}-1.4 C_{D} \lambda \sqrt{U_{i} U_{i}} \varepsilon \\
& \sigma_{\mathrm{k}}=1.0, \sigma_{\varepsilon}=1.3, \quad \mathrm{C}_{\varepsilon 1}=1.44, \mathrm{C}_{\varepsilon 2}=1.92
\end{aligned}
$$

The computer code was developed in-house by the authors. The basic equations are discretized by the Finite Volume method and the SIMPLE algorithm is implemented for pressure-velocity coupling. For unsteady terms, a fully implicit scheme is used. The QUICK scheme is applied to the convection terms and the central differencing scheme is used for the diffusion terms in the momentum equations. The $k$ and $\varepsilon$ equations are discretized by a Power-law scheme. Along the bottom and the side walls a "wall function" technique has been applied. The near free surface turbulent dissipation rate is specified as follows:

$\varepsilon_{s}=C_{\mu}^{3 / 4} k_{s}^{3 / 2} /\left(0.4 \Delta Z_{s}\right)$

Where, the suffix $s$ indicates the value at the point adjacent to the free surface and $\Delta Z_{\mathrm{s}}$ is the normal distance from the free surface.

\section{Passive Contaminant Transport Model}

The concentration of a passive scalar can be computed by solving the following transport equation:

$$
\frac{\partial C}{\partial t}+\frac{\partial U_{j} C}{\partial x_{j}}=\alpha \frac{\partial^{2} C}{\partial x_{j} \partial x_{j}}-\frac{\partial}{\partial x_{j}}\left(\overline{u_{j}^{\prime} c^{\prime}}\right)
$$

Where, $C$ is the scalar concentration, $\alpha$ is the molecular diffusivity of the scalar $=1.612 \times 10-5$ $\left(\mathrm{cm}^{2} / \mathrm{s}\right)$ for $\mathrm{NaCl}$ at $25^{\circ} \mathrm{C}$ temperature and $\overline{u_{j}^{\prime} c^{\prime}}$ is the scalar flux composed of turbulent flux and the correlation between deviated velocity and concentration from their spatial averages. 


\section{The 2014 International Conference on Marine and Freshwater Environments}

Joint Conferences:

The 2014 Annual Conference of the International Society for Environmental Information Sciences (ISEIS)

The 2014 Atlantic Symposium of the Canadian Association on Water Quality (CAWO)

The 2014 Annual General Meeting and zoth Anniversary Celebration of the Canadian Society for Civil Engineering Newfoundland and Labrador Section (CSCE-NL)

The and International Conference of Coastal Biotechnology (ICCB) of the Chinese Society of Marine Biotechnology and Chinese Academy of Sciences (CAS)

Daly and Harlow (1970) introduced an eddy diffusivity tensor proportional to the Reynolds stress for the scalar flux. The DH model represents an algebraic flux model and gives a general form of the flux vector as a linear combination of the Reynolds stress:

$\overline{u_{i}^{\prime} c^{\prime}}=-C_{c} \tau_{c} \overline{u_{j}^{\prime} u_{m}^{\prime}} \frac{\partial C}{\partial x_{m}}$

Where, $C_{c}$ is the model parameter $(=0.4)$ and, $\tau_{c}$ is the characteristic time-scale $=k / \varepsilon$.

Zero-flux condition is applied along the boundaries. The concentration at the injection point is specified.

\section{Results and Discussions}

The non-linear $k-\varepsilon$ turbulence model coupled with the DH model for solute transport was first validated against the experimental data a test case in Shiono et al. (2003). It is seen that the DH model reproduces a reasonable agreement with the measurements (Jahra, 2011). The turbulent flow model was validated against different flow fields in the previous studies (Jahra et al., 2010, 2011). The following sections contain a brief discussion of the calculated results and the respective experimental data.

\section{Floodplain Fully Covered by Model Vegetation: Case A1}

The laboratory experiments have been carried out for three different cases based on solute injection points described in Table 1. Figure 5 shows the lateral distribution of non-dimensional solute concentration near free surface at $x=1,000 \mathrm{~cm}$ and $1,100 \mathrm{~cm}$, respectively. Here in Case $\mathrm{S} 1$ salt water was injected at $y=91 \mathrm{~cm}$. In the figures $C_{0}$ represents the initial solute concentration. The measured result is compared with the calculated result. The DH model, which takes into account the anisotropy of turbulence shows a reasonable agreement with the measured result although some discrepancy was observed near the bottom of the channel. The spread of solute is attributed to convection due to secondary currents and turbulent diffusion which is clearly perceived in the other case studies. The former cannot be identified clearly in Figure 5 due to diminutive secondary currents in the main channel.
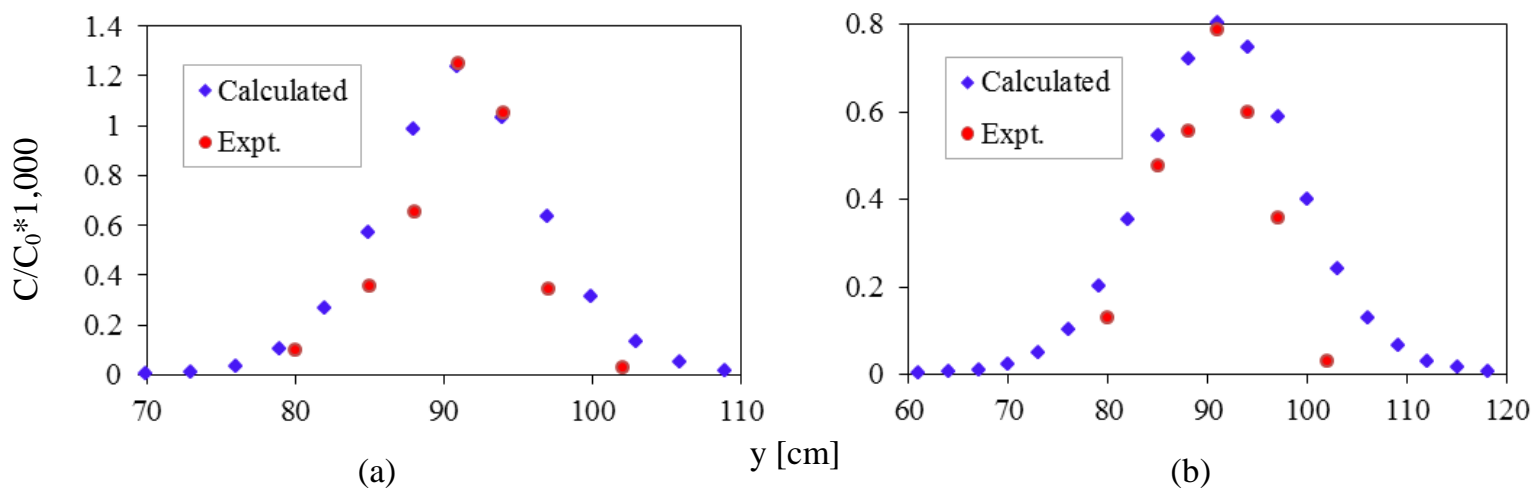

(b)

Figure 5. Contour of solute concentration $\left[\mathrm{C} / \mathrm{C}_{0} * 1000\right]$ in Case $\mathrm{S} 1$ at (a) $x=1,000 \mathrm{~cm}$; (b) $x=1,100 \mathrm{~cm}$. 


\section{The 2014 International Conference on Marine and Freshwater Environments}

Joint Conferences:

The 2014 Annual Conference of the International Society for Environmental Information Sciences (ISEIS)

The 2014 Atlantic Symposium of the Canadian Association on Water Quality (CAWQ)

The 2014 Annual General Meeting and 3oth Anniversary Celebration of the Canadian Society for Civil Engineering Newfoundland and Labrador Section (CSCE-NL)

The and International Conference of Coastal Biotechnology (ICCB) of the Chinese Society of Marine Biotechnology and Chinese Academy of Sciences (CAS)

In Case S2 solute was injected at $y=136 \mathrm{~cm}$, which is located in the main channel, near the interface of the main channel and the vegetated floodplain. Figure 6 shows contour distributions comparing the experimental and the simulated result at $x=1,000 \mathrm{~cm}$. It can be observed that the concentration peak has shifted to the vegetated floodplain and the high concentration region was shifted more in the measured results relative to the calculated ones. The arrow above the free surface indicates the spreading direction of solute concentration and "•" expresses the exact point of solute injection. The secondary cell with clockwise rotation over the vegetated floodplain (Figure 7) plays the dominant role in transporting the solute within the cross-section. The high concentration of solute near the free surface is conveyed from the main channel to the floodplain whereas the solute of low concentration is transported from the floodplain to the main channel near the bed. The same phenomenon can be observed in Case S3 for the solute injection point at $y=138 \mathrm{~cm}$, which is located over the vegetated floodplain, near the interface of the main

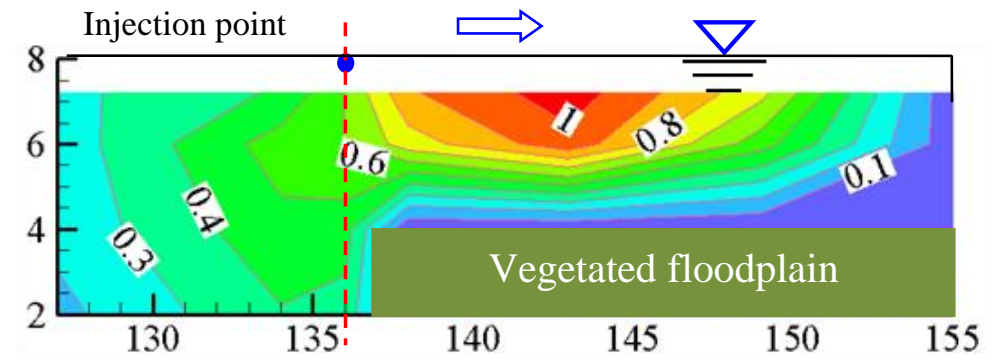

(a)

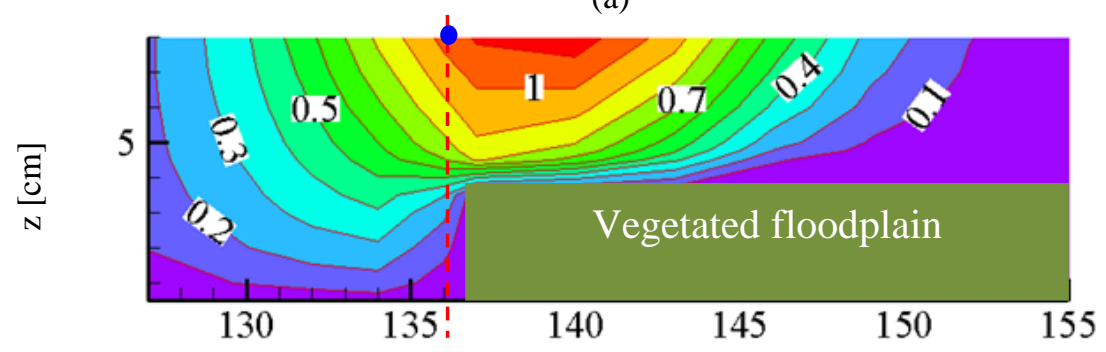

(b)

$\mathrm{y}[\mathrm{cm}]$

Figure 6. Contour of solute concentration $\left[\mathrm{C} / \mathrm{C}_{0} * 1,000\right]$ in Case $\mathrm{S} 2$ at $x=1,000 \mathrm{~cm}$ (a) experimental result; (b) simulated result.

channel and the vegetation zone.

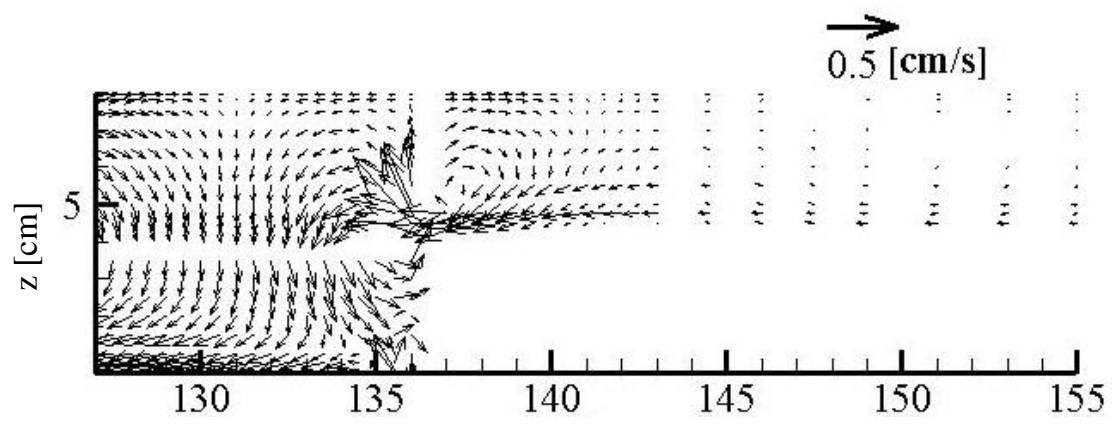




\section{The 2014 International Conference on Marine and Freshwater Environments}

Joint Conferences:

The 2014 Annual Conference of the International Society for Environmental Information Sciences (ISEIS)

The 2014 Atlantic Symposium of the Canadian Association on Water Quality (CAWQ)

The 2014 Annual General Meeting and 3oth Anniversary Celebration of the Canadian Society for Civil Engineering Newfoundland and Labrador Section (CSCE-NL)

The and International Conference of Coastal Biotechnology (ICCB) of the Chinese Society of Marine Biotechnology and Chinese Academy of Sciences (CAS)

Figure 7. Secondary current, $v w$ vector distribution (Case A1).

\section{Floodplain Model Vegetation Belt: Case A2}

The solute concentration distribution is affected by the presence of the vegetation belt over the floodplain located along the interface of the main channel and the floodplain. In Case A2 (floodplain with vegetation belt) salt water was injected at two points at the free surface of the water at $y=136 \mathrm{~cm}$ (Case A2-S4) and $y=148 \mathrm{~cm}$ (Case A2-S5). The first point is located in the main channel near the interface of the floodplain with vegetation belt. The second injection point is located just at the back side of the vegetation belt over the floodplain.

Figure 8 describes the solute distribution when salt water was injected at $y=136 \mathrm{~cm}$. It can be observed that the simulated result underestimates the mixing in the span-wise direction. It is inferred that for complicated flow situations the model parameter $C_{c}$ that controls the scalar flux might need to be tuned. Again, the magnitude of secondary flow in the calculated result is too small to reproduce the measured results accurately. Complicated flow structures due to the vegetation belt can be observed not only in the secondary current distribution but also in largescale turbulence as in Case A2 (Jahra, 2011). A weak clockwise secondary current cell generated within and at the right side of the vegetation belt (Figure 9) restricts the lateral mixing of the solute. Figure 10 shows the solute distribution where salt water was injected just at the right side of the vegetation belt at $y=148 \mathrm{~cm}$. The concentration of the solute was spread within and towards the right side of the vegetation belt due to the distribution of secondary currents. The

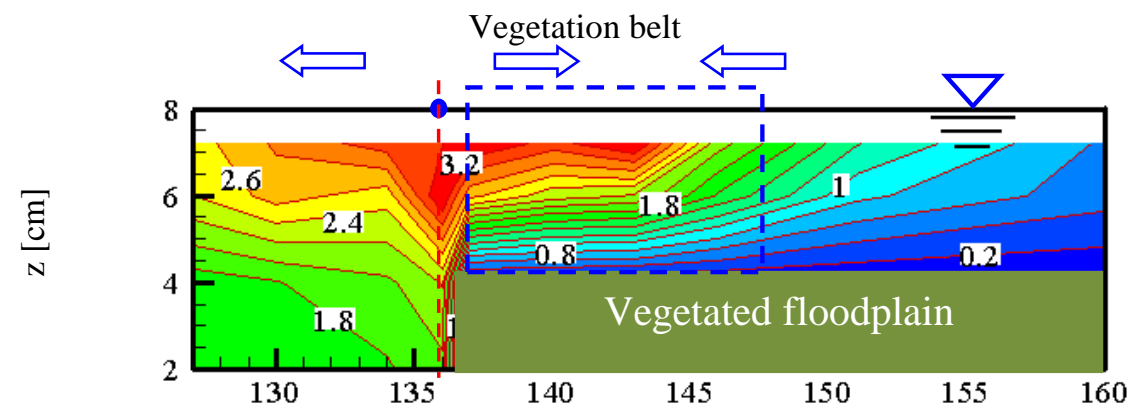

(a)

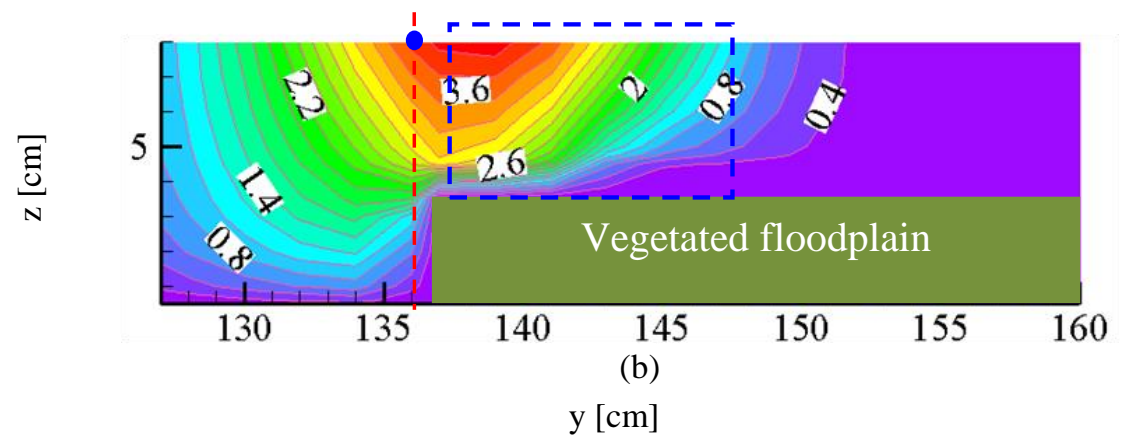

Figure 8. Contour of solute concentration $\left[\mathrm{C} / \mathrm{C}_{0} * 1,000\right]$ in Case $\mathrm{A} 2$ at $x=1,000 \mathrm{~cm}$; (a) experimental result; (b) simulated result.

high concentration zone is shifted towards the right side due to the strong secondary current cell generation at the right side of vegetation belt. 
Joint Conferences:

The 2014 Annual Conference of the International Society for Environmental Information Sciences (ISEIS)

The 2014 Atlantic Symposium of the Canadian Association on Water Quality (CAWQ)

The 2014 Annual General Meeting and zoth Anniversary Celebration of the Canadian Society for Civil Engineering Newfoundland and Labrador Section (CSCE-NL)

The and International Conference of Coastal Biotechnology (ICCB) of the Chinese Society of Marine Biotechnology and Chinese Academy of Sciences (CAS)

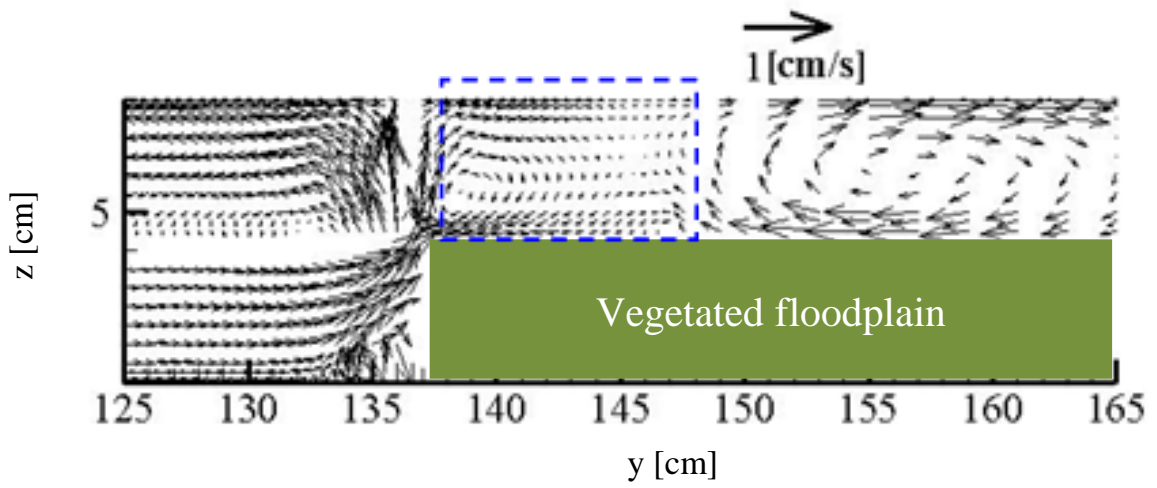

Figure 9. Secondary current, $v w$ vector distribution (Case A2).

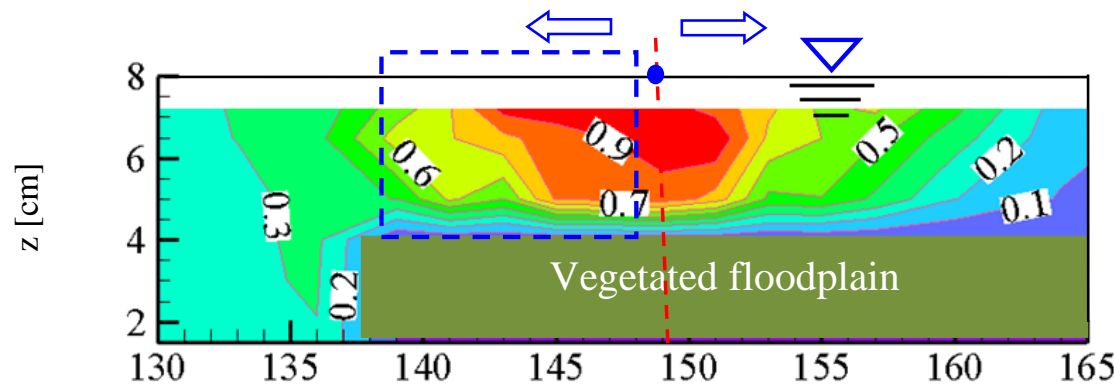

(a)

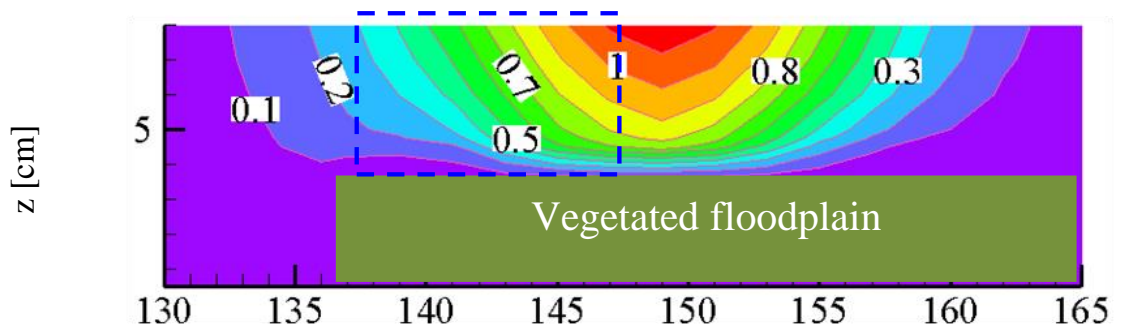

(b)

$\mathrm{y}[\mathrm{cm}]$

Figure 10. Contour of solute concentration $\left[\mathrm{C} / \mathrm{C}_{0} * 1,000\right]$ in Case $\mathrm{A} 2$ at $x=1,100 \mathrm{~cm}$ (a) experimental result; (b) calculated result.

The present model has shown good performance against the experimental data of Shiono et al. (2003) as secondary current is strong due to the absence of floodplain vegetation. It is noted that comparison between the experimental and the calculated results gives an impression that the measured data may have some inaccuracies, which may be due to the larger solute density than the flume water due to the evaporation of alcohol with time.

\section{Conclusions}

Flume experiments were carried out for turbulent open channel flows in the presence of the floodplain vegetation under emergent conditions. Numerical simulations were performed with a non-linear $k-\varepsilon$ model coupled with a vegetation model together with an algebraic flux model for 


\section{The 2014 International Conference on Marine and Freshwater Environments}

\section{Joint Conferences:}

The 2014 Annual Conference of the International Society for Environmental Information Sciences (ISEIS)

The 2014 Atlantic Symposium of the Canadian Association on Water Quality (CAWQ)

The 2014 Annual General Meeting and zoth Anniversary Celebration of the Canadian Society for Civil Engineering Newfoundland and Labrador Section (CSCE-NL)

The and International Conference of Coastal Biotechnology (ICCB) of the Chinese Society of Marine Biotechnology and Chinese Academy of Sciences (CAS)

solute transport. Through comparisons between the calculated results and the measured data by the authors it can be inferred that

- The algebraic flux model by Daly and Harlow, taking into account the anisotropy of turbulence, gives a reasonable agreement with the measured pollutant concentration distribution.

- It is necessary to tune the model parameter $C_{c}$ that controls the scalar flux.

- Secondary currents generated near the free surface enhance the shifting of solute concentration peak from the main channel towards the vegetated floodplain.

- It is also useful to refine the turbulence model to more accurately predict secondary flows which play an important role in solute transfer process.

\section{Acknowledgement}

The authors would like to thank the Government of Japan for providing the first author the MEXT Scholarship, which made this study possible. The corresponding author would also like to express her gratitude to the Oceanic Consulting Corporation for support in publishing this research as an article.

\section{References}

Arnold U., Pasche E. and Rouve G. (1985). Mixing in river of compound cross section, Proc. 21st IAHR Congress, Melbourne, Australia, pp. 168-172.

Daly B.J., Harlow F. H. (1970). Transport equations in turbulence, Phys. Fluids, 13, 2634-2649.

Green S.R. (1992). Modelling turbulent air flow in a stand of widely-spaced trees. PHOENICS Journal of Computational Fluid Dynamics and Application, 5, 294-312.

Jahra F., Yamamoto H., Hasegawa F. and Kawahara Y. (2010). Mean flow and turbulence structure in meandering open channel flows with submerged and emergent vegetation, Proc. Int. Conference on Fluvial Hydraulics (RiverFlow 2010), Braubschweig, Germany, 1, 153-161.

Jahra F., Kawahara Y. and Hasegawa F. (2011). Numerical simulation of turbulent flow in a partially vegetated open channel, Annual Journal of Hydraulic Engineering, JSCE, 55, 193-198.

Jahra F. (2011). Effect of vegetation on flow structure and mass transfer in open channels. Ph.D Dissertation, Department of Civil and Environmental Engineering, Hiroshima University, Japan.

Jaque D.T. and Ball J.E. (1995). Mixing of pollutant concentrations in a compound channel, Proc. 26 ${ }^{\text {th }}$ IAHR Congress, London, UK, Vol. A, pp. 57-62.

Kimura I. and Hosoda T. (2003). A non-linear k- $\varepsilon$ model with realizability for prediction of flows around bluff bodies, International Journal for Numerical Methods in Fluids, 42, 813-837.

Kang H., Choi S. K. and Choi B. (2009). Solute transport in open-channel flows with submerged vegetation, $33^{r d}$ IAHR Congress: Water Engineering for a Sustainable Environment, Vancouver, Canada, pp. 1890-1899.

Lin B. and Shiono K. (1995). Numerical modelling of solute transport in compound channel flows, Journal of Hydraulic Research, 33(6), 773-788.

Naot D., Nezu I., and Kakagawa H. (1996). Hydrodynamic behavior of partly vegetated open-channels, Journal of Hydraulic Engineering, 122(11), 625-633.

Rameshwaran P. and Shiono K. (2007). Quasi two-dimensional model for straight overbank flows through emergent vegetation on floodplains, Journal of Hydraulic Research, 45(3), 302-315.

Sanjou M., Nezu I., Suzuki S. and Itai K. (2010). Turbulence structure of compound open-channel flows with oneline emergent vegetation, 9th International Conference of Hydrodynamics, Shanghai, China, pp.577-581.

Shimizu Y. and Tsujimoto T. (1994). Numerical analysis of turbulent open-channel flow over a vegetation layer using a $k-\varepsilon$ turbulence model, Journal of Hydroscience and Hydraulic Engineering, 11(2), 57-67.

Shiono, K. Scott C. F. and Kearnery D. (2003). Prediction of solute transport in a compound channel using turbulence models, Journal of Hydraulic Research, 41 (3), 247-258.

Wood I. R. and Liang T. (1989). Dispersion in an open channel with a step in the cross-section, Journal of Hydraulic Research, 27(5), 587-601. 
The 2014 International Conference on Marine and Freshwater Environments

Joint Conferences:

The 2014 Annual Conference of the International Society for Environmental Information Sciences (ISEIS)

The 2014 Atlantic Symposium of the Canadian Association on Water Quality (CAWQ)

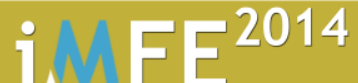

The 2014 Annual General Meeting and zoth Anniversary Celebration of the Canadian Society for Civil Engineering Newfoundland and Labrador Section (CSCE-NL)

The and International Conference of Coastal Biotechnology (ICCB) of the Chinese Society of Marine Biotechnology and Chinese Academy of Sciences (CAS) 